\title{
Correlatos Cognitivos na Aprendizagem da Matemática: uma revisão da literatura
}

\section{Cognitive Correlates in Learning Mathematics: a literature review}

\author{
Carine Franco de Souza* \\ ORCID iD 0000-0003-4355-6722 \\ Neyfsom Carlos Fernandes Matias ${ }^{* *}$ \\ ORCID iD 0000-0001-9064-2282
}

\begin{abstract}
Resumo
O objetivo deste estudo foi o de levantar publicações que investigaram correlatos cognitivos na aprendizagem da Matemática. Foram realizadas pesquisas nas bases de dados Scientific Electronic Library Online (Scielo), Portal de Periódicos Eletrônicos de Psicologia (PePSIC) e Portal de Periódicos da Coordenação de Aperfeiçoamento de Pessoal de Nível Superior (Capes), a partir dos descritores "Matemática", "Matemática and aprendizagem", "desempenho escolar", "desempenho and aritmética" e "aritmética". Para a seleção do material, foram definidos os seguintes critérios: os trabalhos deveriam ser artigos de relatos de pesquisas empíricas, com utilização de delineamento correlacional e apresentação de resultados estatisticamente significativos ( $\mathrm{p}<0,05$ ); os estudos também deveriam ter como amostra estudantes brasileiros que apresentassem desenvolvimento típico. A princípio, a busca resultou em 1.391 trabalhos, dos quais 16 atenderam aos critérios estabelecidos. Entre as habilidades cognitivas investigadas estão as linguísticas, as metacognitivas, a inteligência, a memória, entre outras. A partir dos resultados apresentados, foram apuradas a magnitude das correlações de cada habilidade na aprendizagem da Matemática e a aplicação prática dos achados. Conclui-se que as capacidades investigadas demonstram magnitudes de fraca a forte. Além disso, os pesquisadores apontam a importância de os professores da Educação Básica compreenderem a importância dessas habilidades, o que eles podem fazer para estimulá-las e a necessidade de se promoverem programas voltados para o desenvolvimento das habilidades cognitivas.
\end{abstract}

Palavras-chave: Matemática. Correlatos Cognitivos. Revisão da Literatura. Aritmética.

\begin{abstract}
The objective of this study was to raise publications that investigated cognitive correlates in learning mathematics. Research was carried out in the Scientific Electronic Library Online (Scielo), Electronic Psychology Journals Portal (PePSIC), and Higher Education Personnel Coordination Journals (Capes) databases, from the descriptors: "Matemática", "Matemática and aprendizagem", "desempenho escolar", "desempenho and aritmética", and "aritmética". To select the material, the following criteria were defined: the papers should be articles from empirical research reports, using a correlational design and presenting statistically significant results $(p<0.05)$. The studies should also have been conducted with Brazilian students with typical development. At first, the search resulted in 1.391 works, of which 16 met the criteria. Among the cognitive skills investigated are linguistic, metacognitive, intelligence, memory, among others. From the results presented, we determined the magnitude of the correlations of each skill in learning mathematics and the practical application of the findings. It is concluded
\end{abstract}

\footnotetext{
* Discente do curso de Psicologia da Universidade Federal de São João del-Rei (UFSJ), São João del-Rei, Minas Gerais, Brasil. Endereço para correspondência: Praça Dom Helvécio, 74 - sala 2.20, Dom Bosco, São João delRei, Minas Gerais, Brasil, CEP: 36.301-160. E-mail: carinefancos@gmail.com.

** Doutorado em Psicologia pela Universidade Federal de Minas Gerais (UFMG). Professor do Departamento de Psicologia e do Programa de Pós-Graduação em Psicologia da Universidade Federal de São João del-Rei (UFSJ), São João del-Rei, Minas Gerais, Brasil. Endereço para correspondência: Praça Dom Helvécio, 74 - sala 2.20, Dom Bosco, São João del-Rei, Minas Gerais, Brasil, CEP: 36.301-160.E-mail: neyfsom@ufsj.edu.br.
} 
that the investigated capacities demonstrate weak to strong magnitudes. In addition, researchers point out the importance of primary school teachers understanding the importance of these skills, what they can do to stimulate them, and the need to promote programs aimed at stimulating cognitive skills.

Keywords: Mathematics. Cognitive Correlates. Literature Revision. Arithmetics

\section{Introdução}

Os processos de aprendizagem da Matemática são influenciados por diversos fatores, como habilidades cognitivas e linguísticas, estilos de ensino, estratégias de aprendizagem e características sociais dos estudantes, bem como o nível socioeconômico e as variáveis familiares (OLIVEIRA; NEGREIROS; NEVES, 2015). O desempenho nessa disciplina requer a aquisição integrada de conhecimentos de âmbito específico, sistemas conceituais, princípios de caráter matemático e o desenvolvimento de habilidades cognitivas (ONRUBIA; ROCHEIRA; BARBERÁ, 2004). Os elementos cognitivos e afetivos necessários para a aprendizagem dessa matéria são adquiridos através dos processos de construção social e das mediações culturais. A Matemática não se faz presente apenas no contexto escolar, mas em um cenário mais amplo, que diz respeito aos contextos sociais onde os indivíduos estão inseridos (FALCÃO, 2008; ONRUBIA; ROCHEIRA; BARBERÁ, 2004).

As crianças nascem com o sentido inato do número e potencial para aprender a Matemática, o que demonstra, assim, as interferências dos aspectos biológicos e evolutivos no desenvolvimento das habilidades numéricas (OLIVEIRA; NEGREIROS; NEVES, 2015). Porém essa prontidão não significa que os métodos de ensino não são importantes e, ao mesmo tempo, esta é uma disciplina considerada difícil de assimilar. Não por acaso, há inúmeros questionamentos acerca dos motivos de o desenvolvimento das capacidades matemáticas ser insatisfatório no ambiente escolar (OLIVEIRA; NEGREIROS; NEVES, 2015). Os resultados dos estudantes brasileiros matriculados em escolas públicas, do ano de 2017 , apontam que $56 \%$ dos alunos do $5^{\circ}$ ano e $85 \%$ do $9^{\circ}$ ano mostraram baixa proficiência na resolução de problemas matemáticos (QEDU, 2019). Por isso, é importante identificar os aspectos que interferem nos processos de aquisição de conhecimentos matemáticos, pois assim é possível traçar estratégias para diminuir as dificuldades nessa disciplina.

As habilidades cognitivas estão relacionadas com as características genéticas e as experiências sociais das pessoas (HAASE et al., 2012). Sabe-se que essas habilidades interferem na aprendizagem da Matemática. Alguns dos aspectos cognitivos apontados como mediadores nos processos de aprendizagem dessa disciplina são as abstrações, o processamento fonológico, o cálculo mental, a memória de trabalho, as habilidades visoespaciais, a velocidade 
de processamento e o senso numérico (ALBERGARIA; PONTE, 2008; BECKER, 2014; CARVALHO; PONTE, 2015; CORSO; DORNELES, 2010; CORSO; DORNELES, 2014; HAASE et al., 2012; PIAGET, 1995; SILVA et al., 2015).

O fato de esse campo de pesquisa ser relativamente novo demonstra a importância de se desenvolverem estudos relacionados aos correlatos cognitivos que interferem na aprendizagem da Matemática, a fim de promover ações de caráter interdisciplinar que possam potencializar os processos de ensino e aprendizagem (CORSO; DORNELES, 2010; CORSO; DORNELES, 2014; HAASE et al., 2012). Nesse sentido, buscou-se, por meio de uma revisão bibliográfica, elencar quais são os aspectos cognitivos apontados por pesquisas empíricas como associados com essa disciplina em publicações brasileiras. Assim, este trabalho teve o objetivo de levantar estudos que investigaram a relação entre habilidades cognitivas e aprendizagem da Matemática. Especificamente, buscou-se: 1) apurar quais as habilidades estudadas; 2) identificar de que forma as habilidades cognitivas são apontadas como importantes na aprendizagem da Matemática; 3) pesquisar a magnitude - fraca, moderada e forte (DANCEY; REIDY, 2006) das correlações entre as habilidades cognitivas e o desempenho em Matemática; 4) levantar indicações de práticas para o desenvolvimento e/ou aprimoramento dessas capacidades; 5) apontar questões relacionadas às habilidades cognitivas a serem consideradas na prática docente que podem colaborar com o desempenho matemático.

\section{Método}

Realizou-se uma revisão sistemática da literatura. A fim de alcançar os objetivos propostos, foram consultadas as seguintes bases de dados: Scientific Electronic Library Online (Scielo), Portal de Periódicos Eletrônicos de Psicologia (PePSIC) e Portal de Periódicos da Coordenação de Aperfeiçoamento de Pessoal de Nível Superior (Capes). A busca foi realizada a partir da conjugação dos seguintes descritores: "Matemática", "Matemática and aprendizagem", “desempenho escolar", "desempenho and aritmética" e "aritmética". Em relação aos termos de pesquisa, é pertinente destacar que o descritor "aritmética" foi utilizado por se referir à avaliação da Matemática dentro das pesquisas em psicologia. Isso acontece pelo uso do Teste de Desempenho Escolar (TDE) (STEIN, 1994).

A fim de definir uma estratégia de busca, foram estabelecidos alguns critérios. Para serem incluídos na seleção, os trabalhos deveriam ser relatos de pesquisas empíricas, com utilização de delineamento correlacional (Pearson ou Spearman) e apresentação de resultados estatisticamente significativos $(\mathrm{p}<0,05)$. Esse tipo de delineamento permite a compreensão 
sobre como e quais habilidades cognitivas tem relação com a aprendizagem da Matemática. Os estudos também deveriam ter como amostra estudantes brasileiros que apresentassem desenvolvimento típico (PAPALIA; FELDMAN; MARTORELL, 2013) e que estivessem cursando o Ensino Fundamental. O levantamento bibliográfico foi realizado contemplando trabalhos publicados até o ano de 2018. Optou-se por selecionar apenas textos veiculados em português. A seleção dos artigos e a exclusão das duplicatas ocorreram de forma a não privilegiar nenhuma das bases de dados.

\section{Resultados}

A conjugação dos descritores nas buscas resultou na identificação de 1.391 artigos, conforme indicado na Figura 1. Após a análise dos títulos e resumos, foram excluídos os trabalhos duplicados e os que não se enquadravam nos critérios estabelecidos. Destacaram-se 61 trabalhos, dos quais 24 investigaram a interferência de aspectos sociais na aprendizagem da Matemática, 37 relacionavam a aprendizagem da Matemática com aspectos cognitivos, e havia indícios de que estavam dentro dos critérios estabelecidos. Os autores desta pesquisa realizaram a leitura completa dos artigos.

Por fim, foram excluídos 21 artigos, porque não estavam de acordo com os critérios estabelecidos (avaliação comportamental, não uso de análise de correlação, amostras que não estavam no Ensino Fundamental, entre outros), restando assim, 16 trabalhos. A partir de uma ficha elaborada para registro das informações, preenchida e discutida pelos pesquisadores, foram apurados os seguintes dados: ano das publicações, amostras das pesquisas, anos ou séries escolares em que os estudantes estavam matriculados, a qual rede de ensino (pública ou privada) os participantes estavam vinculados, instrumentos utilizados para a avaliação do desempenho em Matemática, foco do estudo, correlações significativas encontradas $(\mathrm{p}<0,05)$, considerações sobre a possibilidade de aprimorar as habilidades cognitivas e/ou de aplicação prática dos achados. 


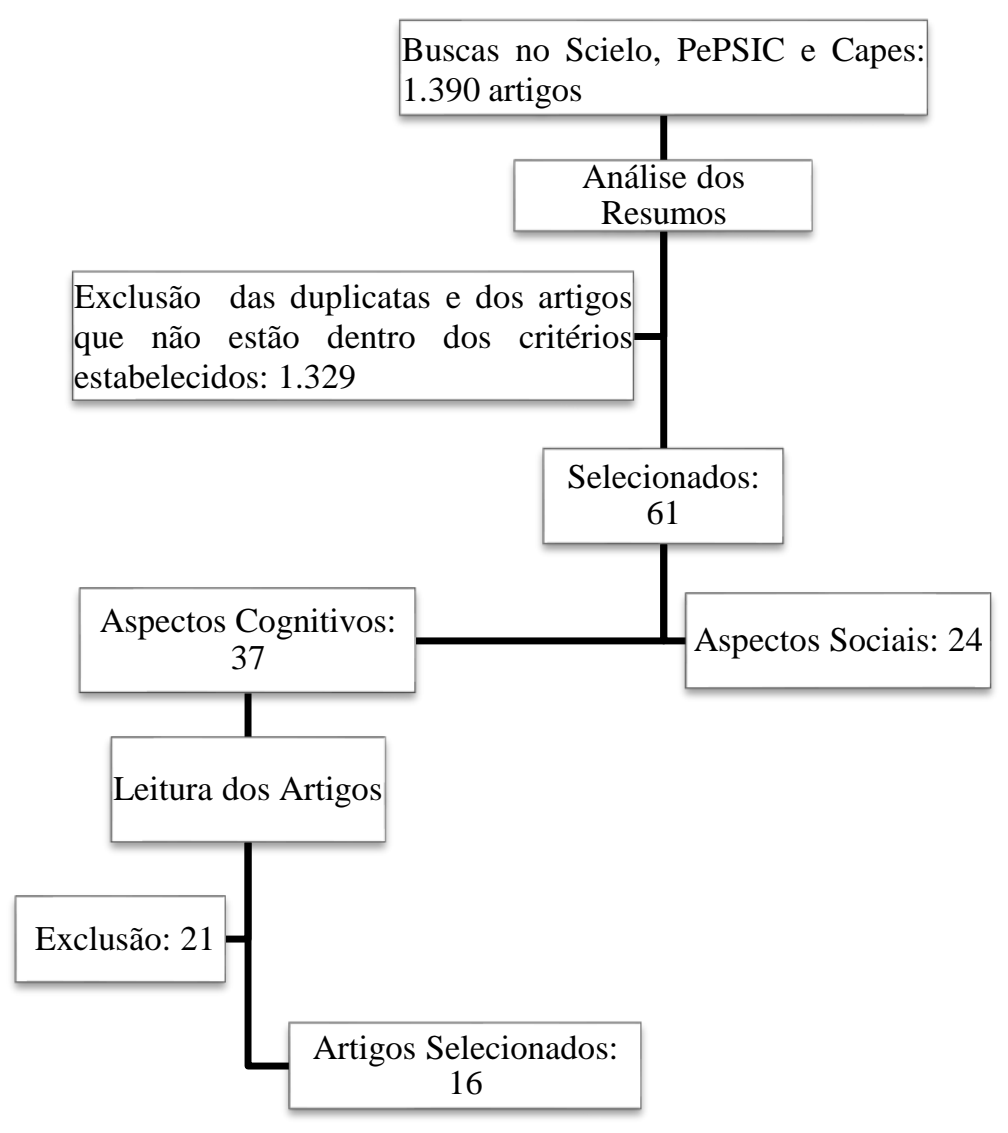

Figura 1 - Fluxograma da seleção dos artigos Fonte: Elaborada pelos autores (2019).

No que diz respeito ao ano de publicação, os artigos encontrados foram publicados a partir de 2008. Conforme consta no Gráfico 1, os anos de 2011, 2015 e 2016 destacam-se pelo maior número de artigos, enquanto 2009, 2013 e 2014 se sobressaem pelo fato de não haver, nesses anos, trabalhos publicados sobre o tema em sintonia com os critérios de investigação.

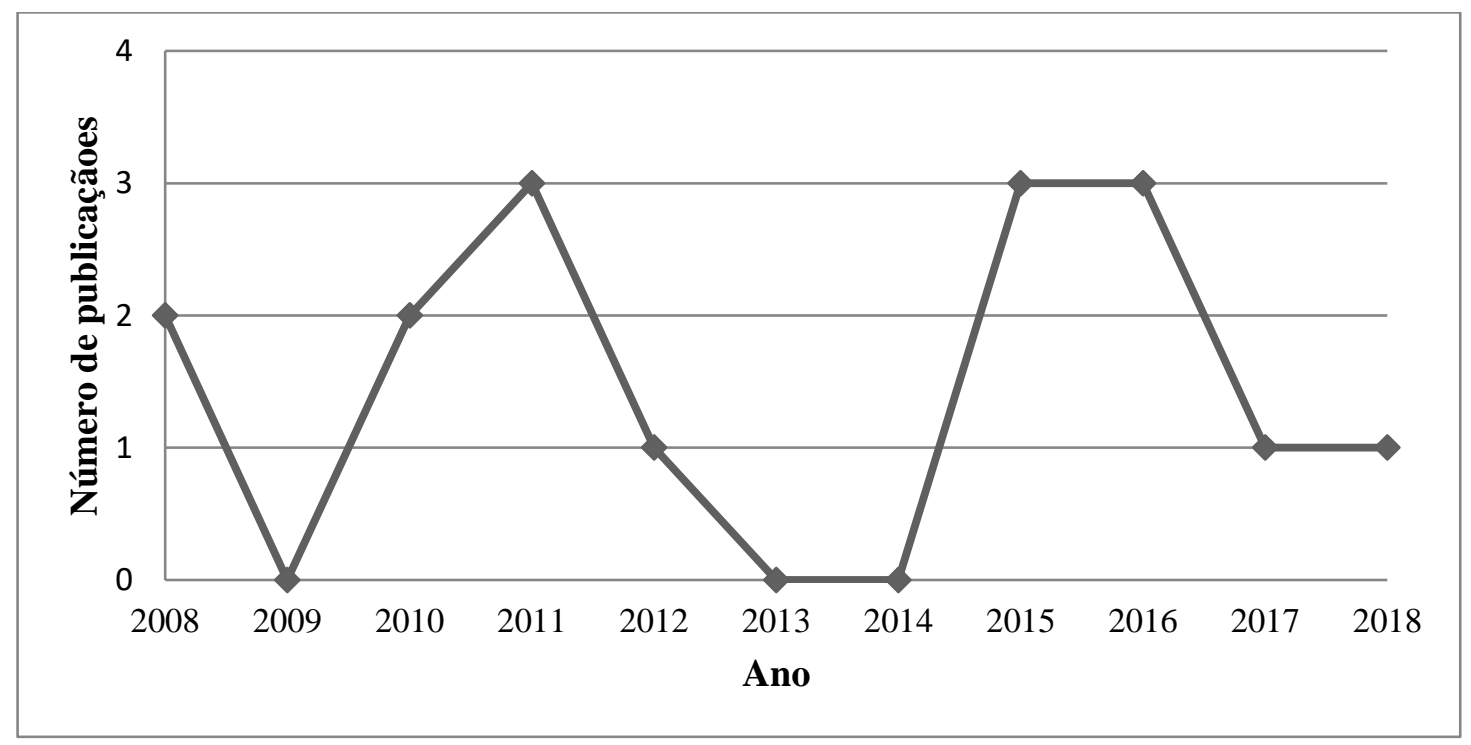

Gráfico 1 - Número de publicações por ano Fonte: Elaborado pelos autores (2019). 
A idade dos participantes das pesquisas estava entre 5 e 15 anos. Entre os trabalhos analisados, 11 pesquisas foram realizadas com estudantes da rede pública, uma com estudantes da rede privada (CONTE; CIASCA; CAPELATTO, 2016) e quatro com estudantes das duas redes (FERREIRA; CONTE; MARTURANO, 2011; MECCA et al., 2016; MECCA et al., 2015; PRADO et al., 2015).

Entre os instrumentos utilizados para a avaliação do desempenho em Matemática, 11 trabalhos aplicaram o subteste de aritmética do TDE (STEIN, 1994). O restante usou a Prova de Aritmética (MECCA et al., 2015; MECCA et al., 2016); as notas escolares dos estudantes (MOL; WECHSLER, 2008); o subteste de cálculos aritméticos do Internacional Dyslexia Test (IDT) (GOLBERT; SALLES, 2010); e a Tarefa de Resolução de Problemas com Equações Algébricas de $1^{\circ}$ grau (TRPEA) (SPERAFICO; DORNELES; GOLBERT, 2015).

A partir do foco dos estudos, realizou-se uma análise de conteúdo que levou à elaboração de seis categorias temáticas. Essa metodologia é uma forma de classificar os itens de sentido, a partir de sua presença ou ausência em determinado tema, com o objetivo de se obter uma imagem simplificada dos dados encontrados (BARDIN, 1977). As categorias elaboradas são apresentadas a seguir, juntamente com os valores das correlações entre as habilidades cognitivas e o desempenho em Matemática, a população estudada, considerando o ano escolar ou série apontada, com o tamanho das amostras e as indicações de promoção dessas capacidades e/ou aplicações práticas dos achados.

a) Habilidades linguísticas: contemplou quatro artigos que apontaram correlações positivas, de fraca a forte, entre essas capacidades e o desempenho em Matemática. Um destes, realizado com alunos cursando a $1^{\mathrm{a}}$ série - Anos Iniciais $(\mathrm{n}=43)$ e $2^{\mathrm{a}}$ série - Anos Iniciais $(\mathrm{n}=$ 45), indicou correlação fraca com a memória fonológica de trabalho $(\mathrm{r}=0,37)$ na $1^{\mathrm{a}}$ série, e correlações moderadas com o processamento fonológico $(\mathrm{r}=0,55)$, consciência fonológica $(\mathrm{r}$ $=0,60)$, leitura $(\mathrm{r}=0,45)$ e escrita $(\mathrm{r}=0,61)$ na $2^{\mathrm{a}}$ série (TENÓRIO; ÁVILA, 2012). O segundo trabalho, com alunos da $2^{\mathrm{a}}$ série - Anos Iniciais $(\mathrm{n}=110)$, indicou correlações moderadas em leitura de palavras isoladas $(\mathrm{r}=0,42)$ e os seguintes componentes da consciência fonológica: rima $(r=0,40)$, aliteração $(r=0,47)$ e exclusão fonêmica $(r=0,41)$ (GOLBERT; SALLES, 2010). Outro, com crianças matriculadas na $2^{\mathrm{a}}$ série - Anos Iniciais $(\mathrm{n}=23)$, apontou correlações moderadas com a consciência fonológica $(r=0,60)$, leitura $(r=0,56)$ e escrita $(r=$ 0,58) (ZUANETTI; SCHNECK; MANFREDI, 2008). Além disso, um trabalho com alunos matriculados entre o $2^{\circ}$ e o $7^{\circ}$ anos - Ensino Fundamental $(n=1.431)$ apontou correlações no desempenho em aritmética e a escrita, a leitura e a fluência verbal semântica. Respectivamente, as correlações foram de moderada $(\mathrm{r}=0,45)$ a forte $(\mathrm{r}=0,83)$, de fraca $(\mathrm{r}=0,37)$ a forte $(\mathrm{r}=$ 
0,79) e de fraca $(\mathrm{r}=0,22)$ a moderada $(\mathrm{r}=0,44)$ (FREITAS; FERREIRA; HAASE, 2010).

As habilidades cognitivas relacionadas à linguagem exercem um importante papel na aprendizagem da Matemática, por exemplo, quando o estudante se depara com representações verbais de números e quantidades (FREITAS; FERREIRA; HAASE, 2010; SILVA et al., 2015). No que diz respeito ao aprimoramento dessas habilidades e aplicação prática dos achados, há diferentes indicações. A possibilidade de atuação da fonoaudiologia junto às escolas é apontada como uma das soluções para aprimorar a relação entre habilidades linguísticas e a aprendizagem da Matemática (TENÓRIO; ÁVILA, 2012). Ademais, aponta-se a necessidade da implantação de programas de Educação Infantil que contemplem aspectos importantes para a aprendizagem da Matemática, como a memória fonológica (GOLBERT; SALLES, 2010). Para além da atuação de áreas específicas vinculadas às habilidades linguísticas, a formação dos professores que atuam com as crianças é indicada no sentido de compreenderem a relação entre linguagem e Matemática. A partir disso, esses profissionais poderão planejar estratégias escolares que colaborem com o desenvolvimento dessas habilidades (FREITAS; FERREIRA; HAASE, 2010).

b) Metacognição: essa categoria engloba quatro estudos que apontam correlações positivas, de fraca a moderada, entre habilidades metacognitivas e o desempenho em Matemática. Uma investigação realizada com crianças cursando entre a $1^{\mathrm{a}}$ e $4^{\mathrm{a}}$ série - Anos Iniciais $(n=50)$ aponta correlações fracas entre a aprendizagem da Matemática e autopercepções de autoconceito $(r=0,29)$ e autoeficácia $(r=0,33)$ (FERREIRA; CONTE; MARTURANO, 2011). O autoconceito também aparece em outros dois trabalhos: o primeiro, realizado com estudantes da $5^{\mathrm{a}}$ e $6^{\mathrm{a}}$ séries - Ensino Fundamental $(\mathrm{n}=58)$, aponta correlações fracas com o autoconceito $(r=0,37)$, especificamente com o autoconceito acadêmico $(r=0,34)$ (CIA; BARHAN, 2008); o segundo, realizado com crianças que estavam entre o $3^{\circ}$ e o $5^{\circ}$ anos - Ensino Fundamental $(n=35)$, encontrou correlações moderada e fraca com o autoconceito pessoal $(r=0,51)$ e o autoconceito social $(r=0,34)$ (CONTE; CIASCA; CAPELATTO, 2016). Por último, um estudo com estudantes do $2^{\circ}$ ano - Anos Iniciais $(\mathrm{n}=50)$ apontou uma correlação fraca entre o desempenho em Matemática e as estratégias de aprendizagem $(r=0,39)$ (PRATES; LIMA; CIASCA, 2016).

A metacognição diz da forma com a qual os estudantes compreendem e regulam os seus próprios processos de aprendizagem (BEBER; SILVA; BONFIGLIO, 2014). A promoção de estratégias de autorregulação valoriza e estimula o desenvolvimento de habilidades e competências promotoras da aprendizagem (BEBER; SILVA; BONFIGLIO, 2014). No que diz respeito ao aprimoramento dessas habilidades e à aplicação prática dos achados, há diferentes 
indicações. Aponta-se a necessidade de as escolas repensarem as atividades voltadas para crianças com dificuldades de aprendizagem: focar apenas questões acadêmicas pode não ser eficaz, pois os problemas socioemocionais também interferem no desempenho escolar (CIA; BARHAN, 2008). Também se sugere que os professores ensinem estratégias de aprendizagem aos alunos, a fim de provocar mudanças no hábito escolar (PRATES; LIMA; CIASCA, 2016).

c) Inteligência: três estudos apontam correlações positivas e moderadas entre essa habilidade e o desempenho em Matemática. Dois trabalhos, um realizado com estudantes do $2^{\circ}$ e $3^{\circ}$ anos - Anos Iniciais $(n=51)$ (MECCA et al., 2016), e outro com estudantes de $6^{\circ}$ a $8^{\circ}$ anos - Ensino Fundamental ( $\mathrm{n}=213$ ) (MECCA et al., 2015), respectivamente, indicam correlações moderadas com a inteligência $(r=0,48)(r=0,51)$. Já um estudo realizado com estudantes do $8^{\circ}$ ano $(n=38)$ encontrou uma correlação moderada $(r=0,63)$ entre essa competência cognitiva e a resolução de problemas algébricos do $1^{\circ}$ grau (SPERAFICO; DORNELES; GOLBERT, 2015).

A inteligência é composta por diferentes habilidades cognitivas, das quais se destacam duas. A primeira é a inteligência fluida que tem grande relevância na aprendizagem da Matemática. Ela é uma capacidade não verbal que diz da possibilidade de resolver problemas com pouco ou nenhum conhecimento prévio, a partir de habilidades como o raciocínio indutivo, sequencial e quantitativo. A segunda é a inteligência cristalizada, que se desenvolve a partir da interação das pessoas com o mundo e depende da aprendizagem (MECCA et al., 2015; MECCA et al., 2016). De certa forma, esta se relaciona com a aprendizagem da Matemática na medida em que o conhecimento nos primeiros anos escolares - por exemplo, a realização das quatro operações básicas - é fundamental para a construção de novos conhecimentos, como a compreensão de uma equação de $1^{\circ}$ grau (SPERAFICO; DORNELES; GOLBERT, 2015). No que diz respeito ao aprimoramento dessas habilidades e aplicação prática dos achados, os autores apontam a importância da realização de atividades que colaborem para o desenvolvimento de habilidades cognitivas, para um melhor desempenho acadêmico (SPERAFICO; DORNELES; GOLBERT, 2015; MECCA et al., 2015).

d) Memória: essa categoria envolve três trabalhos que apontam correlações positivas, de fraca a forte, entre as memórias de trabalho e operacional e a aprendizagem da Matemática. Um estudo realizado com alunos cursando do $4^{\circ}$ ao $7^{\circ}$ ano - Ensino Fundamental $(\mathrm{n}=79)$ investigou a relação entre o desempenho em Matemática e a memória de trabalho em duas tarefas que avaliaram o componente executivo central da memória de trabalho, encontrando correlações fracas: tarefa com informação não numérica $(r=0,26)$ e tarefa com informação numérica $(\mathrm{r}=0,33)(\mathrm{CORSO}, 2018)$. Outro trabalho, com alunos da $1^{\mathrm{a}}$ e $2^{\mathrm{a}}$ séries - Anos Iniciais 
$(\mathrm{n}=30)$, trata da memória de trabalho, especificamente a memória auditiva por número, indicando uma correlação fraca $(r=0,37)$ (MOL; WECHSLER, 2008). O terceiro artigo investigou a relação entre a memória operacional e a aprendizagem da Matemática em estudantes matriculados entre a $3^{\mathrm{a}}$ e $4^{\mathrm{a}}$ séries - Anos Iniciais $(\mathrm{n}=30)$, apontando correlações de moderada a forte entre o desempenho em Matemática e dois componentes, o de esboço visoespacial $(r=0,54$ e $r=0,47)$ e o executivo central para informações visuais $(r=0,52$ e $r=$ $0,56)$ e o componente executivo central relacionado a informações verbais $(r=0,50$ e $r=0,75)$ (SILVA; SANTOS, 2011).

A memória constitui-se como um conjunto de capacidades cognitivas que permite armazenar informações por longos ou curtos períodos de tempo (SILVA; SANTOS, 2011). A memória de trabalho, por exemplo, é responsável por resgatar as informações prévias e eventos armazenados na memória de longo prazo e por processá-los durante uma atividade cognitiva, como a resolução de problemas matemáticos (CARVALHO; PONTE, 2015; CORSO, 2018). No que diz respeito ao aprimoramento dessas habilidades e à aplicação prática dos achados, há diferentes indicações. Sugere-se a avaliação neuropsicológica para identificar crianças com discalculia do desenvolvimento e outras potenciais comorbidades (SILVA; SANTOS, 2011). Além disso, destaca-se a importância de conhecer as características cognitivas dos estudantes que têm relação com a aprendizagem da Matemática, pois esse conhecimento auxilia o professor na aplicação de novas estratégias de ensino (MOL; WECHSLER, 2008) e na elaboração de currículos (CORSO, 2018).

e) Senso numérico: essa categoria contemplou dois artigos que apontaram correlações positivas, de fraca a moderada, entre esse aspecto cognitivo e a aprendizagem da Matemática. O primeiro estudo, realizado com alunos cursando do $4^{\circ}$ ao $7^{\circ}$ ano - Ensino Fundamental (n $=$ 79), relaciona a aprendizagem da Matemática com o senso numérico, apontando uma correlação moderada $(\mathrm{r}=0,56)(\mathrm{CORSO}, 2018)$. O outro estudo, realizado com alunos do $4^{\mathrm{o}}$ ano - Anos Iniciais $(\mathrm{n}=62)$, indica uma correlação fraca entre a aprendizagem dessa disciplina e a habilidade de subtização e estimativa $(r=0,38)$ (PRADO et al., 2015).

O senso numérico refere-se à forma como as crianças lidam com os números, a sua facilidade, flexibilidade e compreensão dos significados e das ideias relacionadas a eles. Um senso numérico pouco desenvolvido ocasiona problemas no desenvolvimento de habilidades fundamentais da fluência Matemática (CORSO; DORNELES, 2010). No que diz respeito ao aprimoramento dessas habilidades e à aplicação prática dos achados, vale ressaltar que conhecer os aspectos que interferem na aprendizagem da Matemática pode auxiliar os professores na construção de currículos adequados às demandas dos alunos (CORSO, 2018). 
f) Integração visuomotora: um estudo realizado com alunos do $2^{\circ}$ ano - Anos Iniciais $(n=77)$ apontou uma correlação positiva fraca $(r=0,27)$ entre essa capacidade e o desempenho em Matemática (PEREIRA; ARAÚJO; BRACCIALLI, 2011). A integração visuomotora refere-se à habilidade de conseguir traduzir de maneira harmoniosa, a partir de movimentos motores, aquilo que se percebe visualmente. Esse tipo de integração é importante para a aprendizagem escolar em razão de ela ter bases visuomotoras. Em relação à Matemática, destaca-se a sua importância na escrita numérica (PEREIRA; ARAÚJO; BRACCIALLI, 2011). Quanto ao aprimoramento dessa capacidade e à aplicação prática dos achados, o artigo aponta a importância de se acompanhar a aptidão motora das crianças que estão em idade escolar como uma ação preventiva (PEREIRA; ARAÚJO; BRACCIALLI, 2011).

Essas categorias foram elaboradas a partir da literatura que aborda os aspectos cognitivos na aprendizagem da Matemática (CORSO, 2018; MECCA et al., 2015; PRATES; LIMA; CIASCA, 2016; PEREIRA; ARAÚJO; BRACCIALLI, 2011; TENÓRIO; ÁVILA, 2012) e pela leitura e discussão dos trabalhos pelos autores deste artigo. Como é possível observar, a partir da forma como os dados foram apresentados, os resultados significativos dos estudos foram usados na construção das categorias. Desta forma, a categorização indicou correlações de fraca a forte entre habilidades linguísticas, integração visuomotora, inteligência, memória, metacognição, senso numérico e a aprendizagem da Matemática. A maioria das investigações abordou o desempenho nessa disciplina a partir do subteste aritmética do TDE (STEIN, 1994), sendo isso um traço predominante nos aspectos metodológicos. Não foi destacada outra hegemonia nas questões abordadas nos artigos.

\section{Discussão}

A Educação no Brasil enfrenta diversos desafios que passam pelas condições sociais da população, implementação de políticas públicas, condição econômica dos governos e relação entre as características individuais dos estudantes (AGUIAR; ORTIGÃO, 2012; VINHA; KARINO; LAROS, 2016). Na presente oportunidade, buscou-se abordar parte dessas condições, com o enfoque nos correlatos cognitivos da aprendizagem da Matemática. Ou seja, teve-se a intenção de apurar estudos que abordaram as correlações entre esses dois aspectos. $\mathrm{O}$ método de revisão sistemática da literatura auxilia na busca dos trabalhos, de maneira a encontrar um maior número de artigos, de forma organizada e com menor risco de a busca ser enviesada pela perspectiva dos autores (COSTA; ZOLTOWSKI, 2014).

Devido ao fato de o Estado brasileiro se destacar por baixos índices no desempenho 
escolar (AGUIAR; ORTIGÃO, 2012), esperava-se encontrar inúmeros trabalhos que apontassem correlações significativas entre as habilidades cognitivas e a aprendizagem da Matemática. No entanto, não foram encontrados muitos artigos sobre esse tema, visto que, em um universo de 1.391 artigos, 37 tratavam de habilidades cognitivas e, destes, 16 destacam interferências significativas $(\mathrm{p}<0,05)$ entre os fatores investigados. Apesar do número baixo, os resultados encontrados apontam que a aprendizagem da Matemática é abordada a partir da relação com diferentes habilidades cognitivas e destacam aspectos importantes a serem debatidos.

A Educação, bem como os problemas de aprendizagem, constitui-se como uma área que demanda trabalhos interdisciplinares. Entre os diversos saberes que a estudam, a Medicina tem se destacado, indicando a necessidade de esses aspectos serem abordados a partir da medicalização (COLLARES; MOYSÉS, 2012). Desta forma, há necessidade de se levantarem outras maneiras de atuação sobre os problemas de aprendizagem, assim como de se compreender como os aspectos cognitivos se relacionam com o desempenho em Matemática. Os resultados deste estudo apontam que, no Brasil, esse tipo de investigação é recente, considerando-se que os trabalhos selecionados foram publicados a partir de 2008. Além disso, em 2009, 2013 e 2014 não foram veiculados trabalhos com correlações significativas entre os aspectos cognitivos e a aprendizagem da Matemática nas bases de dados investigadas.

Uma possível explicação para esse fato pode ser a adoção do critério de que somente trabalhos com resultados com significância estatística $(p<0,05)$ seriam selecionados. Essa opção partiu do pressuposto da necessidade de se pautar em evidências científicas na construção desta revisão. No que se refere aos participantes das investigações, nota-se o predomínio da rede pública como lócus de pesquisa. Apenas um trabalho contou com alunos exclusivamente da rede privada (CONTE; CIASCA; CAPELATTO, 2016). Uma hipótese a ser considerada para entender essa predominância é a de que as instituições privadas não demonstram disposição para colaborar com pesquisas. Ainda em relação às amostras, observa-se que o número de participantes nos estudos é baixo. Isso se deve à logística que os instrumentos usados demandam para aplicação, sendo que a maioria deles é administrada de forma individual. Há um reflexo dessa abordagem na maneira como a Matemática é compreendida nos estudos.

A partir da forma como o desempenho em Matemática foi avaliado, predominantemente pelo subteste de aritmética do TDE (STEIN, 1994), observa-se a compreensão de que ser bom nessa disciplina, significa conseguir realizar cálculos. Apenas um trabalho contemplou aspectos para além da aritmética, abordando o desempenho algébrico dos estudantes (SPERAFICO; DORNELES; GOLBERT, 2015). Isso é algo a ser analisado, considerando que as provas de 
vestibulares e o Exame Nacional do Ensino Médio (Enem) vão além do ato de calcular ao propor a avaliação da Matemática. Essas avaliações são elaboradas a partir de questões com enunciados que exigem a capacidade de interpretação de textos e avaliam também a álgebra (BRASIL, 2019). Nesse sentido, é preciso considerar se a maneira pela qual o desempenho em Matemática abordado nas pesquisas examinadas seria a adequada. Não que o cálculo não seja pertinente, mas, dessa forma, contempla-se apenas parte do problema. Uma possível explicação para essa questão é o fato de que são poucos os instrumentos psicopedagógicos padronizados para a avaliação da Matemática no Brasil. Soma-se a isso a desatualização do TDE, até a realização dos estudos apurados na presente oportunidade, sobretudo, com o que é solicitado nas diferentes avaliações da Educação. O baixo número de instrumentos de avaliação da Matemática indica uma das diversas dificuldades de se investigar o desempenho nessa matéria. Ao se considerarem os aspectos cognitivos, observa-se que os desafios nessa área se destacam.

As seis categorias que resumem os principais achados dos trabalhos que compuseram o corpus desta revisão apontam que a aprendizagem da Matemática não ocorre de maneira isolada e depende de diferentes habilidades cognitivas, destacando-se a dificuldade de aprender Matemática. Dentre os aspectos linguísticos, constata-se que as habilidades fonológicas se sobressaem nos estudos (GOLBERT; SALLES, 2010; TENÓRIO; ÁVILA, 2012; ZUANETTI; SCHNECK; MANFREDI, 2008) como capacidades importantes no desempenho em Matemática. Há de se considerar que esse aspecto tem papel importante na aquisição da leitura e da escrita (MECCA et al., 2015). Não por acaso, existem indicativos de que a dificuldade em Matemática implica também dificuldades na leitura, e o inverso pode ser verdadeiro (GOLBERT; SALLES, 2010; MECCA et al., 2015; TENÓRIO; ÁVILA, 2012; ZUANETTI; SCHNECK; MANFREDI, 2008). Esse fato indica que a avaliação do desempenho em Matemática deve considerar como o estudante se destaca em tarefas de outras disciplinas, por exemplo, em Língua Portuguesa. Desta forma, o olhar do professor sobre o aluno, sobretudo nos primeiros anos de escolarização, deve ir além do que ele apresenta na Matemática.

Nesse quesito de ultrapassar os limites dos números é importante que os docentes considerem a importância de ensinar aos estudantes estratégias que colaborem com o desempenho em Matemática. Ou seja, na medida em que isso aconteça, há a colaboração no desenvolvimento da metacognição. Isso pode acontecer a partir da indicação pelo professor de quais são as potencialidades dos estudantes e da constatação de que eles conseguem e podem elaborar formas para melhorar o seu aprendizado. O estudante tem mais disposição para aprender quando percebe o seu potencial intelectual de forma positiva (FERREIRA; CONTE; MARTURANO, 2011). Pelo seu caráter exato e devido ao fato de o algoritmo sempre conduzir 
a uma resposta correta (ALBERGARIA; PONTE, 2008), na Matemática é evidente o quanto se está aprendendo porque não há resultados diferentes na solução de uma expressão, por exemplo. Assim, os estudantes acompanharão o seu desenvolvimento e isso promoverá ou não um senso de autoeficácia. Em função dessa questão, a correlação entre esse aspecto e o desempenho matemático é positiva (CIA; BARHAN, 2008; CONTE; CIASCA; CAPELATTO, 2016).

No que se refere à categoria inteligência, destaca-se a vinculação entre aspectos desenvolvimentais, no caso da inteligência fluida, e o papel das interações, dos aspectos culturais e dos processos de ensino/aprendizagem na evolução da inteligência cristalizada (SCHELINI, 2006). A relação entre essa habilidade e a aprendizagem da Matemática ocorre de maneira interdependente, na medida em que o desenvolvimento das habilidades numéricas nos primeiros anos escolares é importante para a elaboração de novos conhecimentos (SPERAFICO; DORNELES; GOLBERT, 2015). Por outro lado, a aprendizagem do conteúdo matemático interfere na capacidade de resolver problemas novos. Ou seja, há interferências dessa habilidade na inteligência cristalizada por ela ser dependente do conhecimento adquirido no decorrer da vida.

$\mathrm{Na}$ categoria memória, destaca-se o foco na memória de trabalho nos três artigos que estudaram o tema. Essa habilidade é importante no desempenho em Matemática por ser fundamental na solução de tarefas relacionadas a contar, a resolver operações e ao cálculo mental (CARVALHO; PONTE, 2015; CORSO, 2018). A aprendizagem está vinculada diretamente aos processos de memorização, que podem se relacionar com as estratégias metacognitivas. Isso significa que é possível ensinar aos estudantes estratégias de memorização e maneiras para que eles possam identificar como conseguem armazenar melhor as informações matemáticas. Desta forma, observa-se a interlocução entre duas habilidades cognitivas que podem ser desenvolvidas na sala de aula pelos professores.

Quanto à categoria senso numérico, constata-se que essa habilidade contempla aspectos aprimorados no decorrer do desenvolvimento (CORSO, 2018) e aspectos inatos, como a subtização e a estimativa (PRADO et al., 2015). Independentemente da sua origem, percebe-se uma relação direta entre a aprendizagem das habilidades numéricas e o desenvolvimento do senso numérico, ou seja, na medida em que ocorre a aprendizagem da Matemática, há implicações no desenvolvimento dessa capacidade cognitiva. Nesse sentido, há uma interação entre aspectos cognitivos e desempenho matemático.

A última categoria abordada trata da integração visuomotora. Essa habilidade tem implicações no desempenho da Matemática à medida que o estudante necessita reproduzir a escrita de números nos processos de aprendizagem (PEREIRA; ARAÚJO; BRACCIALLI, 
2011). Desse modo, é importante que a escola esteja atenta ao desenvolvimento psicomotor da criança e à forma como ela representa, através da escrita, aquilo que é compreendido (PEREIRA; ARAÚJO; BRACCIALLI, 2011). Assim, é possível que, ao identificar alguma dificuldade, a criança seja encaminhada para um profissional capacitado.

Quanto à força das correlações, como era de se esperar, vai de fraca a forte. A partir dessas informações, identifica-se que o componente executivo central relacionado a informações verbais da memória, à leitura e à escrita se destacou devido a sua maior magnitude. Ainda assim, isso não nos permite dizer que essas habilidades são as mais importantes. Os estudos de correlação não necessariamente apontam efeitos das habilidades cognitivas em si, mas sim indicam a importância de se pensar nelas na prática docente e no desenvolvimento de programas voltados para a aprendizagem da Matemática.

A partir do estudo realizado, ressalta-se a importância da interdisciplinaridade nos contextos de ensino da Matemática. As correlações com as habilidades linguísticas e a forma como algumas avaliações, como o Enem, são construídas, apontam que o ensino focado apenas na aprendizagem do cálculo não é o suficiente. Recursos como programas que incentivem a leitura e a escrita podem auxiliar os alunos a desenvolverem essas habilidades. Entender quais aspectos cognitivos interferem na aprendizagem da Matemática pode auxiliar o docente na forma pela qual as aulas são planejadas, para que o ensino de estratégias, como de aprendizagem e memorização, colabore com o desempenho dos alunos. Além disso, intervenções de outras áreas de conhecimento, como a Fonoaudiologia e a Psicologia, podem auxiliar os alunos diante de dificuldades relacionadas a essas habilidades cognitivas.

\section{Considerações finais}

A presente revisão da literatura buscou sistematizar o que as pesquisas têm considerado ao trabalhar os correlatos cognitivos na aprendizagem da Matemática. Habilidades linguísticas, metacognitivas, inteligência, memória, senso numérico e integração visuomotora são apontadas como interferentes nos processos de aprendizagem da Matemática. É importante a compreensão desses aspectos por parte das equipes escolares, pois entende-se que algumas dificuldades de aprendizagem podem estar relacionadas ao desenvolvimento dessas habilidades.

Convém destacar que a amostra analisada é apenas um recorte das pesquisas sobre o tema em questão e a prática docente do ensino da Matemática deve também levar em consideração os aspectos sociais e afetivos. Diante disso, ressalta-se a importância de novos estudos que abranjam esses e outros aspectos. As buscas privilegiaram fontes que 
contemplavam mais trabalhos da Psicologia do que outras áreas. Isso se caracteriza como uma limitação do estudo. No entanto, essa opção se deu pelo fato de que os correlatos cognitivos são os principais objetos de estudo de investigadores de áreas da Psicologia. Para pesquisas futuras, sugere-se a consulta a outras bases de dados e que se considerem diferentes características das amostras, como as demais fases escolares e estudantes com desenvolvimento atípico, além de apurar as contribuições dos trabalhos com resultados não significativos. Espera-se que o estudo aqui relatado colabore não só com as informações de como os correlatos cognitivos se relacionam com a aprendizagem da Matemática, mas também com a prática dos docentes que atuam no Ensino Fundamental.

\section{Referências}

Obs. As referências assinaladas com um asterisco apontam os estudos que compuseram o corpus da revisão.

AGUIAR, G. S.; ORTIGÃO, M. I. Letramento em matemática: um estudo a partir dos dados do PISA 2003. Bolema, Rio Claro, v. 26, n. 42a, p. 1-22, abr. 2012.

ALBERGARIA, I. S.; PONTE, J. P. Cálculo mental e calculadora. In: CANAVARRO, A. P.; MOREIRA, D.; ROCHA, M. I. (ed.). Tecnologias e Educação Matemática. Lisboa: SEM-SPCE, 2008. p. 98-109.

BARDIN, L. Análise de conteúdo. Lisboa: Edições 70, 1977.

BEBER, B.; SILVA, E.; BONFIGLIO, S. U. Metacognição como processo da aprendizagem. Revista Psicopedagogia, São Paulo v. 31, n. 95, p. 144-151, 2014.

BECKER, F. Abstração pseudo-empírica e reflexionante: significado epistemológico e educacional. Revista Eletrônica de Psicologia e Epistemologia Genéticas, Marília, v. 6, p. 104-128, nov. 2014.

BRASIL. Matriz de Referência ENEM. 2019. Brasília: Instituto Nacional de Estudos e Pesquisas Educacionais Anísio Teixeira, 2019. Disponível em:

http://download.inep.gov.br/download/enem/matriz_referencia.pdf. Acesso em: 14 set. 2019.

CARVALHO, R.; PONTE, J. P. Cálculo mental com números racionais: representações mentais dos alunos. In: ENCONTRO DE INVESTIGAÇÃO EM EDUCAÇÃO MATEMÁTICA, 2015, Bragança. Atas... Bragança: SPIEM, 2015. p. 69-83.

*CIA, F.; BARHAM, E. J. Estabelecendo relação entre autoconceito e desempenho acadêmico de crianças escolares. Psico, Porto Alegre, v. 39, n. 1, p. 21-27, jan./mar. 2008.

COLLARES, C. A. L.; MOYSÉS, M. A. A. A medicalização do não-aprender-na-escola e a invenção da infância anormal. In: CHACON, M.; MARIN, M. J. S. (org.). Educação e saúde de grupos especiais. São Paulo: Cultura Acadêmica, 2012. p. 185-197.

*CONTE, G.; CIASCA, S. M.; CAPELATTO, I. V. Relação entre autoconceito e autocontrole comparados ao desempenho escolar de crianças do ensino fundamental. Revista Psicopedagia, São Paulo, v. 33, n. 102, p. 225-234, 2016. 
CORSO, L. V.; DORNELES, B. V. Senso numérico e dificuldades de aprendizagem na matemática. Revista Psicopedagogia, São Paulo, v. 27, n. 83, p. 298-309, 2010.

CORSO, L. V.; DORNELES, B. V. A velocidade de processamento e as dificuldades de aprendizagem na aritmética. Estudos e Pesquisas em Psicologia, Rio de Janeiro, v. 14, n.3, p. 949-966, dez. 2014.

*CORSO, L. V. Memória de trabalho, senso numérico e desempenho em aritmética. Psicologia: Teoria e Prática, São Paulo, v. 20, n. 1, p. 155-167, abr. 2018.

COSTA, A. B.; ZOLTOWSKI, A. P. C. Como escrever um artigo de revisão sistemática. In: KOLLER, S. H.; COUTO, M. C. P. de P.; VON HOHENDORFF, J. (ed.). Manual de produção científica. Porto Alegre: Penso, 2014. p. 55-70.

DANCEY, C. P.; REIDY, J. Estatística sem matemática para psicologia usando SPSS para Windows. 3. ed. Tradução de Lorí Vialí. Porto Alegre: Artmed, 2006.

FALCÃO, J. T. R. Psicologia da Educação Matemática: uma introdução. Belo Horizonte: Editora Autêntica, 2008.

*FERREIRA, A. A.; CONTE, K. M.; MARTURANO, E. M. Meninos com queixa escolar: autopercepções, desempenho e comportamento. Estudos de Psicologia, Campinas, v. 28, n. 4, p. 443451, dez. 2011.

*FREITAS, N. L.; FERREIRA, F. O.; HAASE, V. G. Linguagem e matemática: estudo sobre relações entre habilidades cognitivas linguísticas e aritméticas. Ciências \& Cognição, Rio de Janeiro, v. 15, n. 3, p. 111-125, dez. 2010.

*GOLBERT, C. S.; SALLES, J. F. Desempenho em leitura/escrita e em cálculos aritméticos em crianças de 2 ${ }^{a}$ série. Psicologia Escolar e Educacional, Campinas, v. 14, n. 2, p. 203-210, dez. 2010.

HAASE, V. G. et al. Heterogeneidade cognitiva nas dificuldades de aprendizagem da matemática: Uma revisão bibliográfica. Psicologia em Pesquisa, Juiz de Fora, v. 6, n. 2, p. 139-150, dez. 2012.

*MECCA, T. P. et al. Relação entre habilidades cognitivas não-verbais e variáveis presentes no contexto educacional. Psicologia Escolar e Educacional, Maringá, v. 19, n. 2, p. 329340, ago. 2015.

*MECCA, T. P. et al. Relação entre habilidades cognitivas de processamento visual e inteligência fluida com o desempenho em aritmética. Psico, Porto Alegre, v. 47, n. 1, p. 35-45, 2016.

*MOL, D. A. R.; WECHSLER, S. M. Avaliação de crianças com indicação de dificuldades de aprendizagem pela bateria Woodcock-Johnson III. Psicologia Escolar e Educacional, Campinas, v. 12, n. 2, p. 391-399, dez. 2008.

OLIVEIRA, M. F.; NEGREIROS, J. G. M.; NEVES, A. C. Condicionantes da aprendizagem da matemática: uma revisão sistêmica da literatura. Educação e Pesquisa, São Paulo, v. 41, n. 4, p. 10231037, mai. 2015.

ONRUBIA, J.; ROCHEIRA, M. J.; BARBERÁ, E. O Ensino e a aprendizagem da matemática: uma perspectiva psicológica. In: COLL, C.; MARCHESI, A.; PALACIOS, J. (org.). Desenvolvimento psicológico e educação: psicologia da educação escolar. 2. ed. Porto Alegre: Artmed, 2004. p. 329333. v. 2.

PAPALIA, D. E.; FELDMAN, R. D.; MARTORELL, G. Desenvolvimento humano. 12. ed. Porto Alegre: Artmed, 2013. 
*PEREIRA, D. M.; ARAÚJO, R. C. T.; BRACCIALLI, L. M. P. Análise da relação entre a habilidade de integração visuo-motora e o desempenho escolar. Revista Brasileira Crescimento

Desenvolvimento Humano, São Paulo, v. 21, n. 3, p. 808-817, 2011.

PIAGET, J. Abstração reflexionante: relações lógico-aritméticas e ordem das relações espaciais. Tradução de Fernando Becker e Petronilha G. da Silva. Porto Alegre: Artes Médicas, 1995.

*PRADO, P. S. T. et al. Desempenho de alunos do quarto ano em testes de subitização e estimativa e no Teste de Desempenho Escolar (TDE). Temas em Psicologia, Ribeirão Preto, v. 23, n. 1, p. 1$14,2015$.

*PRATES, K. C. R.; LIMA, R. F.; CIASCA, S. M. Estratégias de aprendizagem e sua relação com o desempenho escolar em crianças do Ensino Fundamental I. Revista Psicopedagia, São Paulo, v. 33, n. 100, p. 19-27, 2016.

QEDU. Aprendizado dos alunos: Brasil. 2019. Disponível em:

https://www.qedu.org.br/brasil/aprendizado. Acesso em: 21 set. 2019.

SCHELINI, P. W. Teoria das inteligências fluida e cristalizada: início e evolução. Estudos de Psicologia, Natal, v. 11, n. 3, p. 323-332, dez. 2006.

SILVA, J. B. L. et al. Processamento fonológico e desempenho em aritmética: uma revisão da relevância para as dificuldades de aprendizagem. Temas em Psicologia, Ribeirão Preto, v. 23, n. 1, p. 157-173, 2015.

*SILVA, P. A.; SANTOS, F. H. Discalculia do desenvolvimento: avaliação da representação numérica pela ZAREKI-R. Psicologia: Teoria e Pesquisa, Brasília, v. 27, n. 2, p. 169-177, jun. 2011.

*SPERAFICO, Y. L. S.; DORNELES, B. V.; GOLBERT, C. S. Competência cognitiva e resolução de problemas com equações algébricas do $1^{\circ}$ grau. Bolema, Rio Claro, v. 29, n. 51, p. 333-348, abr. 2015.

STEIN, L. M. TDE: teste de desempenho escolar: manual para aplicação e interpretação. São Paulo: Casa do Psicólogo, 1994.

*TENORIO, S. M. P. C. P.; ÁVILA, C. R. B. de. Processamento fonológico e desempenho escolar nas séries iniciais do ensino fundamental. Revista CEFAC, São Paulo, v. 14, n. 1, p. 30-38, fev. 2012.

VINHA, L. G. A.; KARINO, C. A.; LAROS, J. A. Factors Associated with Mathematics Performance in Brazilian Basic Education. Psico-USF, Itatiba, v. 21, n. 1, p. 87-100, abr. 2016.

*ZUANETTI, P. A.; SCHNECK, A. P. C.; MANFREDI, A. K. S. Consciência fonológica e desempenho escolar. Revista CEFAC, São Paulo, v. 10, n. 2, p. 168-174, 2008.

Submetido em 04 de Novembro de 2019. Aprovado em 05 de Agosto de 2020. 